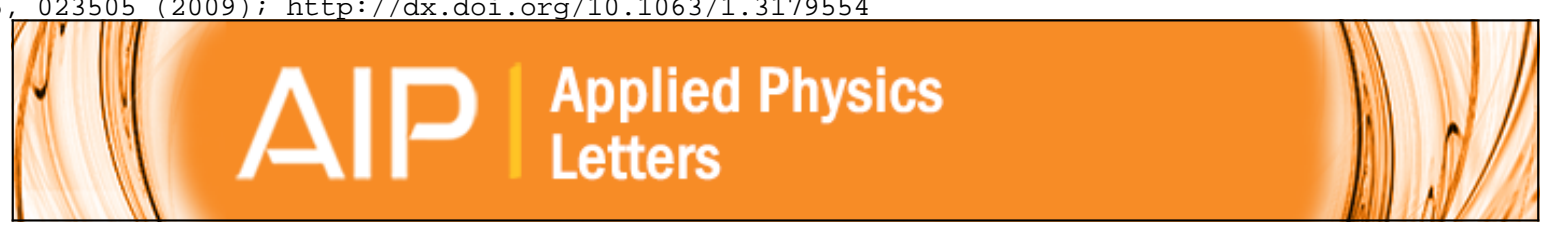

Layer-by-layer self-assembled conductor network composites in ionic polymer metal composite actuators with high strain response

Sheng Liu, Reza Montazami, Yang Liu, Vaibhav Jain, Minren Lin, James R. Heflin, and Q. M. Zhang

Citation: Applied Physics Letters 95, 023505 (2009); doi: 10.1063/1.3179554

View online: http://dx.doi.org/10.1063/1.3179554

View Table of Contents: http://scitation.aip.org/content/aip/journal/apl/95/2?ver=pdfcov

Published by the AIP Publishing

Over 700 papers \&
presentations on

multiphysics simulation new now

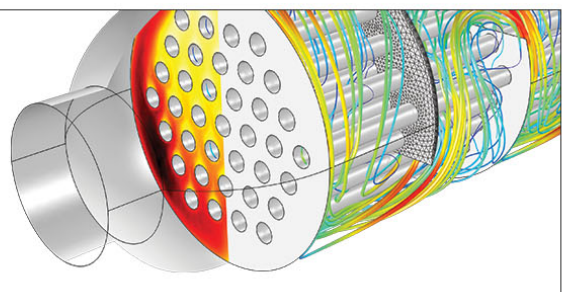




\title{
Layer-by-layer self-assembled conductor network composites in ionic polymer metal composite actuators with high strain response
}

\author{
Sheng Liu, ${ }^{1}$ Reza Montazami, ${ }^{2, a)}$ Yang Liu, ${ }^{1}$ Vaibhav Jain, ${ }^{3}$ Minren Lin, ${ }^{4}$ James R. Heflin, ${ }^{5}$ \\ and Q. M. Zhang ${ }^{1,4}$ \\ ${ }^{1}$ Department of Electrical Engineering, The Pennsylvania State University, University Park, \\ Pennsylvania 16802, USA \\ ${ }^{2}$ Department of Materials Science and Engineering, Virginia Tech, Blacksburg, Virginia 24061, USA \\ ${ }^{3}$ Macromolecular Science and Engineering, Virginia Tech, Blacksburg, Virginia 24061, USA \\ ${ }^{4}$ Materials Research Institute, The Pennsylvania State University, University Park, \\ Pennsylvania 16802, USA \\ ${ }^{5}$ Department of Physics, Virginia Tech, Blacksburg, Virginia 24061, USA
}

(Received 6 April 2009; accepted 18 June 2009; published online 16 July 2009)

\begin{abstract}
We investigate the electromechanical response of conductor network composite (CNC) fabricated by the layer-by-layer (LbL) self-assembly method. The process makes it possible for CNCs to be fabricated at submicron thickness with high precision and quality. This CNCs exhibits high strain $\sim 6.8 \%$ under $4 \mathrm{~V}$, whereas the $\mathrm{RuO}_{2} / \mathrm{Nafion} \mathrm{CNCs}$ exhibit strain $\sim 3.3 \%$. The high strain and submicron thickness of the LbL layers in an ionic polymer metal composite (IPMC) yield large and fast actuation. The response time of a $26 \mu \mathrm{m}$ thick IPMC with $0.4 \mu \mathrm{m}$ thick LbL CNCs to step voltage of 4 V is 0.18 s. (C) 2009 American Institute of Physics. [DOI: 10.1063/1.3179554]
\end{abstract}

Electroactive polymers (EAPs), which generate large electromechanical actuation while operated under low voltage (approximately a few volts), are attractive since they have the potential to be easily integrated with microelectronic controlling circuits to perform complex functions. ${ }^{1-7}$ Early works on the Pt-electroded Nafion is an example of EAPs exhibiting a relatively large bending actuation under a few volts of applied voltage, which attracted a great deal of attention. ${ }^{4-7}$ Since then, much research effort has been devoted to this class of EAPs with the objective of further improving the electromechanical performance properties such as the actuation speed, strain level, and efficiency. ${ }^{4-14}$

Figure 1(a) illustrates schematically such an ionic polymer bending actuator in which the accumulation and depletion of cations at the cathode and anode, respectively, create bending of the ionic polymer membrane under an electrical signal. Experimental results have indicated that a high population of the excess charges at the electrodes is highly desirable in order to generate high electromechanical actuation. To realize that, porous electrodes which offer large electrode areas in contact with ionomers are often utilized in this class of actuators. ${ }^{4-14}$ One widely investigated ionic polymer metal composite (IPMC) uses a chemical reduction method to deposit precious metals to Nafion (a perfluorosulfonated ionomer developed by DuPont) membrane surfaces, in which nanosized metal particles penetrate into the Nafion membrane to form porous electrodes. ${ }^{5-7,11,14}$ More recently, a direct assembly method in which conductive nanoparticles were mixed with a Nafion (or an ionomer) solution and this mixture is directly deposited on the Nafion (or an ionomer) membrane to fabricate IPMC was developed. ${ }^{8,9,12,13}$ The direct assembly method to fabricate IPMCs is attractive because it allows for the use of a broad range of nanosized conductors for the electrodes such as carbon nanotubes. ${ }^{13}$ It also simplifies the fabrication process of IPMCs and en-

${ }^{a)}$ Electronic mail: rezam@vt.edu. hances actuator manufacturing repeatability., ${ }^{8,9,12,13}$ Illustrated in Fig. 1(b) is a typical bimorph (bending) actuator thus developed, which in general has a three layer structure, i.e., two porous composite electrode layers, which are referred to as the conductor network composite (CNC) and generate strain under applied voltage, separated by a neat ionomer layer.

In this paper, we investigate a class of $\mathrm{CNC}$ fabricated by a direct assembly method to form IPMC bimorph actuators in which the CNC electrodes were deposited onto the Nafion membrane via a layer-by-layer (LbL) self-assembly process. The CNC made of the LbL composite exhibits a very large strain $(\sim 6.8 \%)$ as deduced from the bending actuation of the bimorph actuator. Furthermore, an LbL CNC allows for precise control of the $\mathrm{CNC}$ thickness on the nanometer scale. By utilizing this LbL CNC, IPMC bimorph actuators with the CNC layer thickness $\sim 0.4 \mu \mathrm{m}$ display a large bending actuation with a fast actuation time $(\sim 0.18 \mathrm{~s})$. For comparison, we also fabricated IPMC bimorph actuators using $\mathrm{RuO}_{2} / \mathrm{Nafion}$ nanocomposite as the $\mathrm{CNCs}$ by the direct assembly method. $\mathrm{RuO}_{2} / \mathrm{Nafion}$ nanocomposite ionic polymer conductor network composites (IPMCs) have been investigated extensively and have shown high strain response among the IPMCs developed. ${ }^{4-13}$

For the IPCNCs investigated in this paper, the commercial Nafion film NR-211 of thickness $25 \mu \mathrm{m}$ was chosen as

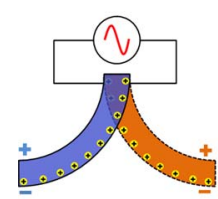

(a)

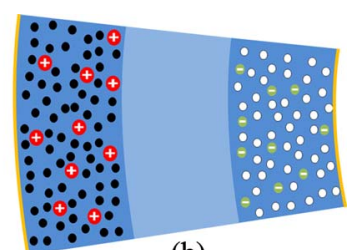

(b)
FIG. 1. (Color online) (a) Schematic of an ionomer bending actuator under an electric signal. (b) Schematic of a CNC/ionomer/CNC (C/I/C) three layer IPMC bimorph actuator (the Au electrode layers are also shown). 

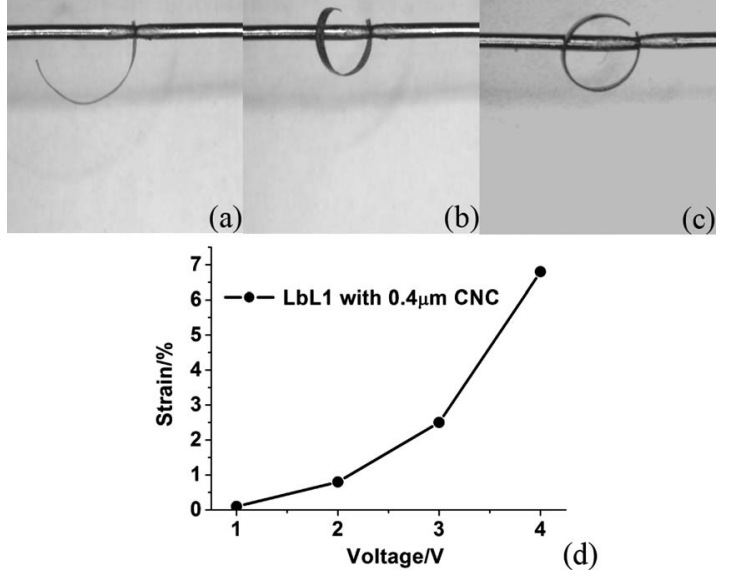

FIG. 2. Photographic images of the bending actuation under $4 \mathrm{~V}$. (a) The 100 bilayers LbL composite actuator; (b) the 200 bilayers LbL composite actuator; (c) the $3 \mu \mathrm{m} \mathrm{RuO}_{2} / \mathrm{Nafion}$ composite actuator; and (d) the 100 bilayers LbL CNC strain $S_{10}^{c}$ vs applied voltage.

the ionomer layer in Fig. 1(b). The LbL composite electrodes are fabricated by immersing the Nafion film into two oppositely charged solutions alternately, which contain anionic gold nanopaticles $(\sim 2 \mathrm{~nm}$ diameter, Purest Colloids, Inc.) and the polycation poly(allylamine hydrochloride), respectively. ${ }^{15-17}$ The composites grow via the electrostatic attraction between polyelectrolyte or nanoparticles in solution and the oppositely charged substrate. The LbL CNCs, comprising 100 and 200 LbL bilayers, have electrode thicknesses of 0.4 and $0.8 \mu \mathrm{m}$, respectively. For each IPCNC, there are $\mathrm{CNC}$ electrodes on each of the two surfaces and hence the total IPCNC thickness is 25.8 and $26.6 \mu \mathrm{m}$, respectively. The IPMCs with these two CNC layer thicknesses are referred to as LbL1 and LbL2 in the paper. $\mathrm{RuO}_{2} / \mathrm{Nafion}$ composites are prepared using the direct assembly method. ${ }^{13}$ $\mathrm{RuO}_{2}$ nanoparticles purchased from Alfa Aesar with 13-19 $\mathrm{nm}$ diameters are mixed with $20 \%$ Nafion dispersion from Aldrich. The mixture is sonicated before being sprayed onto the Nafion film surface. After spraying, films are transferred to a vacuum oven to rid them of solvent and are then ready for actuator fabrication. The $\mathrm{RuO}_{2} / \mathrm{Nafion}$ ionomer composite thickness was $3 \mu \mathrm{m}$ and hence the total thickness of the IPMC is $31 \mu \mathrm{m}$.

After composites are grown on the neat Nafion membrane, actuators are fabricated by soaking samples with 40 wt \% ionic liquid 1-ethyl-3-methylimidazolium trifluoromethanesulfonate (EMI-Tf) and depositing $50 \mathrm{~nm}$ thick gold leaves as external electrodes on the two sides of the samples. Several other room temperature ionic liquids were also tested, and it was found that IPCNCs with EMI-Tf generate the highest strain response. The lateral dimensions of all the actuators are $1 \mathrm{~mm}$ in width and $8 \mathrm{~mm}$ in length.

The time resolved bending strain of the actuators was recorded by a charge coupled device video camera attached to a probe station. A step voltage was applied to generate bending strain and the maximum applied voltage is $4 \mathrm{~V}$. The elastic modulus of each layer along the film surface direction in the IPCNC was characterized using a setup specifically designed to measure the elastic modulus of soft materials. In this setup, the specimen is fixed at two ends and a displacement transducer was used to generate strain in the specimen and the corresponding stress (force/cross section area) was measured by a load cell. In this study, the elastic modulus of

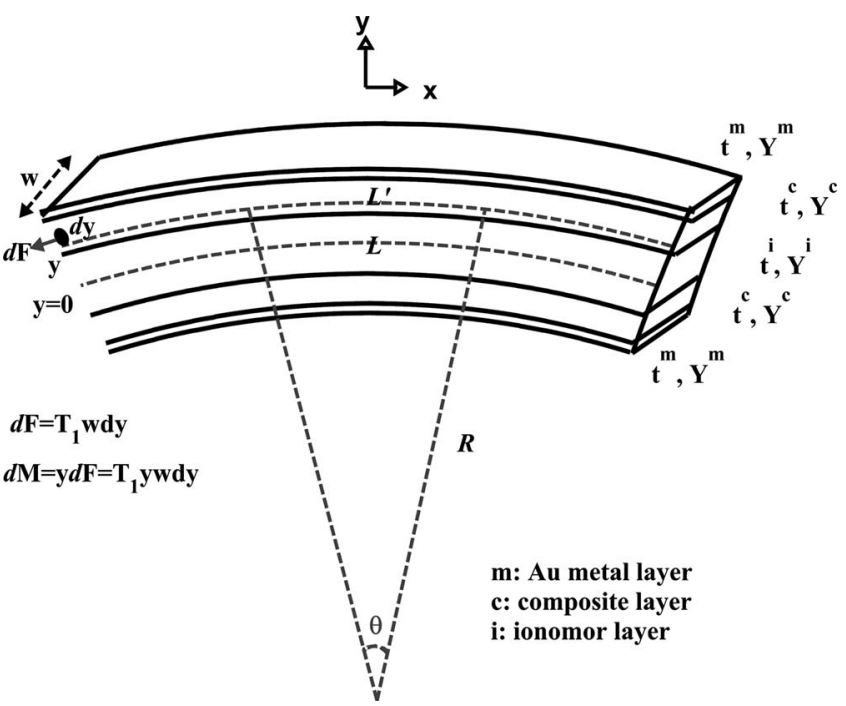

FIG. 3. Schematic of the five layer bending actuator used for the derivation of the relation between the bending radius of curvature $R$ and initial strain $S_{10}^{c}$ in the CNC layers [Eq. (3)]. $Y$ is the Young's modulus and $t$ is the layer thickness. Superscipts $m, c$, and $i$ indicate Au metal layer, conductor composite, and ionomer layer, respectively.

the Nafion film with 40 wt \% EMI-Tf was measured first $(=50 \mathrm{MPa})$. Then the elastic modulus of the specimen with CNC deposited to Nafion membrane was characterized from which the elastic modulus of the CNC layer is deduced $\left(Y=0.4 \mathrm{GPa}\right.$ for LbL CNCs and $=0.3 \mathrm{GPa}$ for $\mathrm{RuO}_{2}$ nanocomposite CNCs). The elastic modulus of the five layer IPCNC swollen with EMI-Tf was also measured from which the elastic modulus of the $\mathrm{Au}$ layer was deduced $(=20 \mathrm{GPa})$.

Presented in Fig. 2 are the bending actuation of the LbL1, LbL 2, and $\mathrm{RuO}_{2}$ /Nafion IPCNC. In general, for such a bending actuator, a thicker $\mathrm{CNC}$ layer will generate larger bending if the intrinsic strain in the CNC layer $\left(S_{10}^{c}\right)$ is the same. In these bimorph actuators, the bending actuation is generated by the strains in the CNC layers. In order to extract the intrinsic strain in the CNC layer $\left(S_{10}^{c}\right)$ which is a material property of $\mathrm{CNC}$ layers, we derive the relationship between the bending radius of curvature $R$ and the strain $S_{10}^{c}$ along the film surface in the CNC layers (see Fig. 3). In the bending actuators, the actual strain $S_{1}^{c}$ is reduced from $S_{10}^{c}$ due to the stresses from the ionomer layer and $\mathrm{Au}$ film, i.e.,

$$
S_{1}^{c}=S_{10}^{c}+s_{11}^{c} T_{1}^{c}
$$

where $s_{11}^{c}$ is the elastic compliance and $T_{1}^{c}$ is the stress along the film surface (see Fig. 3). It is noted that $S_{10}^{c}$ is a property of the CNC material and will not change, for example, with the various layer thicknesses in the bimorph actuators [Fig. 1(b)]. On the other hand, actual strain in the CNC layers $S_{1}^{c}$ will change with the bimorph configuration, ranging from near zero to $S_{10}^{c}$. For the bending actuator in Fig. 3, it can be assumed that all the other stress components are very small compared with $T_{1}^{c}$ and can be approximated as zero. ${ }^{18,19}$ Further, it can be derived that the strain $S_{1}$ is related to the radius of the curvature $R$ as 19 


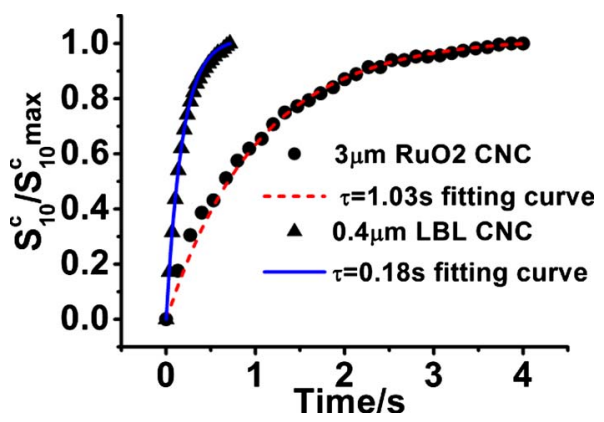

FIG. 4. (Color online) The actuator response as a function of time under a step voltage for the $\mathrm{LbL} 1\left(25.8 \mu \mathrm{m}\right.$ total thickness) and $\mathrm{RuO}_{2} / \mathrm{Nafion} \mathrm{CNC}$ based IPCNC (31 $\mu \mathrm{m}$ total thickness).

$$
S_{1}=\frac{L^{\prime}-L}{L}=\frac{(y+R) \theta-R \theta}{R \theta}=\frac{y}{R}=\kappa y .
$$

From the equilibrium conditions, when there is no external force or moment, the total moment $M$ and total force $F$ of the bending actuator satisfy $\int d F=0$ and $\int d M=0$. Assuming the strain $S_{10}^{c}$ in the two CNC layers in the bending actuator is the same in magnitude with opposite sign (one expanding and one contracting), $\int d F=0$ is true for any $S_{10}^{c}$ value. Therefore, integrating over the thickness for $\int d M=0$, initial strain in the CNC layer $S_{10}^{c}$ can be derived as

$$
\frac{1}{R}=-\frac{Y^{c}\left(t^{i} t^{c}+t^{c^{2}}\right) S_{10}^{c}}{Y^{m}\left(\frac{2 t^{m^{3}}}{3}+2 t^{m}\left(t^{c}+\frac{t^{i}}{2}\right)^{2}+t^{m^{2}}\left(2 t^{c}+t^{i}\right)\right)+Y^{c}\left(\frac{2 t^{c^{3}}}{3}+t^{c} \frac{t^{i^{2}}}{2}+t^{c^{2}} t^{i}\right)+Y^{i} \frac{t^{i^{3}}}{12}}
$$

The meaning of each parameter in Eq. (3) is illustrated in Fig. 3. From the radius of the curvature measured from several LbL1 and LbL2 bending actuators, the strain $S_{10}^{c}$ is derived to be $6.8 \%$ under $4 \mathrm{~V}$ step voltage. In comparison, the strain $S_{10}^{c}$ in the $\mathrm{RuO}_{2} \mathrm{CNC}$ layer is $3.3 \%$ under the same voltage. In Fig. 2(d), we present $S_{10}^{c}$ of LbL CNCs as a function of applied voltage. The results here show that the LbL CNCs can generate much higher strain under low applied voltage compared with other EAPs reported. ${ }^{2,20}$

The bimorph bending actuation speed was also characterized for these actuators. Presented in Fig. 4 is the strain $S_{10}^{c}$ (in reduced unit) as a function of time for the LbL1 and the IPMC with $3 \mu \mathrm{m} \mathrm{RuO} /$ Nafion nanocomposite as CNC. The data is fitted with $S=S_{0}[1-\exp (-t / \tau)]$, which yields a $\tau=0.18 \mathrm{~s}$ for the LbL1 and $\tau=1.03 \mathrm{~s}$ for the IPMC with $3 \mu \mathrm{m} \mathrm{RuO}_{2}$ /Nafion nanocomposite electrodes. The fast actuation speed of LbL1 is due to the thin CNC layer which is $0.4 \mu \mathrm{m}$ compared with $3 \mu \mathrm{m}$ for the $\mathrm{RuO}_{2} / \mathrm{Nafion}$ composite electrodes. In conclusion, the results presented indicate the potential of LbL self-assembled CNCs as a class of EAPs for large actuations under low applied voltage.

The authors thank Ralph Colby and Wenjuan Liu for many stimulating discussions regarding the work. This material is based upon work supported in part by the U.S. Army Research Office under Grant No. W911NF-07-1-0452 Ionic Liquids in Electro-Active Devices ILEAD MURI. Two of the authors (S.L. and R.M.) contributed equally to this work.
${ }^{1}$ R. H. Baughman, C. Cui, A. A. Zakhidov, Z. Iqbal, J. N. Barisci, G. M. Spinks, G. G. Wallace, A. Mazzoldi, D. De Rossi, A. G. Rinzler, O. Jaschinski, S. Roth, and M. Kertesz, Science 284, 1340 (1999).

${ }^{2}$ Y. Bar-Cohen and Q. M. Zhang, MRS Bull. 33, 173 (2008).

${ }^{3}$ E. W. H. Jager, E. Smela, and O. Inganas, Science 290, 1540 (2000).

${ }^{4}$ M. Shahinpoor, Y. Bar-Cohen, J. Simpson, and J. Smith, Smart Mater. Struct. 7, R15 (1998).

${ }^{5}$ K. Oguro, Y. Kawami, and H. Takenaka, J. Micromachine Soc. 5, 27 (1992)

${ }^{6}$ K. Onishi, S. Sewa, K. Asaka, N. Fujiwara, and K. Oguro, Electrochim. Acta 46, 737 (2000).

${ }^{7}$ M. Shahinpoor, J. Intell. Mater. Syst. Struct. 6, 3 (1995).

${ }^{8}$ B. J. Akle, M. D. Bennett, and D. J. Leo, Sens. Actuators, A 126, 173 (2006).

${ }^{9}$ M. D. Bennett and D. J. Leo, Sens. Actuators, A 115, 79 (2004).

${ }^{10}$ I. S. Park, K. Jung, D. Kim, S. M. Kim, and K. J. Kim, MRS Bull. 33, 3 (2008).

${ }^{11}$ S. Nemat-Nasser and Y. Wu, J. Appl. Phys. 93, 5255 (2003).

${ }^{12}$ B. J. Akle, D. Leo, M. Hickner, and J. McGrath, J. Mater. Sci. 40, 3715 (2005).

${ }^{13}$ B. J. Akle and D. J. Leo, J. Intell. Mater. Syst. Struct. 19, 8 (2008).

${ }^{14}$ D. Kim, K. J. Kim, Y. Tak, D. Pugal, and I. S. Park, Appl. Phys. Lett. 90, 184104 (2007).

${ }^{15} \mathrm{G}$. Decher, Science 277, 1232 (1997).

${ }^{16}$ P. T. Hammond, Adv. Mater. (Weinheim, Ger.) 16, 1271 (2004).

${ }^{17}$ V. Jain, H. M. Yochum, R. Montazami, and J. R. Heflin, Appl. Phys. Lett. 92, 033304 (2008).

${ }^{18}$ J. M. Herbert, Ferroelectric Transducers and Sensors (Gordon and Breach, New York, 1982).

${ }^{19}$ Q. M. Wang and L. E. Cross, Ferroelectrics 215, 187 (1998).

${ }^{20}$ J. D. W. Madden, N. A. Vandesteeg, P. A. Anquetil, P. G. A. Madden, A. Takshi, R. Z. Pytel, S. R. Lafontaine, P. A. Wieringa, and I. W. Hunter, IEEE J. Ocean. Eng. 29, 706 (2004). 